\title{
Análisis genético y propiedades virulentas de cepas de Escherichia coli aisladas desde infección vaginal
}

\author{
Olga Lobos, Andrés Padilla y Carlos Padilla
}

\section{Genetic analysis and virulent properties of Escherichia coli strains isolated from vaginal infection}

Background: Escherichia coli is able to produce different infections in humans. It pathogenicity in the female genital tract is unknown. Objective: To determine the presence of virulence genes (VG) in E. coli strains isolated from the female genital tract. Material and Methods: $146 \mathrm{E}$. coli strains isolated as monomicrobial cultures from vaginal infections were genetically characterized by search of $h l y, i u c C$, afa, fimH, neuC, sfa/foc, cnFl, papC, usp, and ibeA VG. Studies were performed by means PFGE and PCR. Results: Genetic analysis of the strains showed two groups with a similarity of approximately $80 \%$. The similarity genetic intragroup was approximately $95 \%$. The results showed strains with a high number of VG and the most common were $c n f l$ and fimH. The $a f a$ gene was not detected. Were identified eight VG combinations and the most common was papC $+h l y+i u c C+$ $a f a-n e u C-f i m H+s f a / f o c+c n f 1+u s p+i b e A-$ Discussion: The studied strains are concentrated in two genetic groups. Most of the strains contain a great number of VG present in E. coli isolated from extraintestinal infections. Conclusion: It is important to develop new research strategies in this area, to deepen the phylogenetic knowledge of these strains and confirm their true role in vaginal infection.

Key words: Escherichia coli, vaginal infections, virulence genes, pulsed field gel electrophoresis, PCR.

Palabras clave: Escherichia coli, infecciones vaginales, genes de virulencia, electroforesis de campo pulsado, RPC.

\section{Introducción}

$\mathrm{L}$ as infecciones vaginales son un problema común en medicina clínica y la causa más recurrente para visitar un ginecólogo ${ }^{1-3}$. Las consecuencias de las infecciones vaginales son variables, ocasionando desde daño sicológico en las mujeres, hasta abortos espontáneos y bajo peso al nacer de los neonatos ${ }^{4,5}$. En el mundo nacen prematuramente 13 millones de niños, lo que representa aproximadamente $10 \%$ de los nacimientos; $30 \%$ de éstos se encuentran asociados a cuadros infecciosos ${ }^{6}$.

Los tres tipos más importantes de infecciones vaginales son las candidiasis, tricomoniasis y la vaginosis bacteriana, constituyendo aproximadamente $90 \%$ de las enfermedades infecciosas que afectan el tracto genital femenino ${ }^{7}$. Escherichia coli ha sido frecuentemente aislada desde infecciones vaginales y actualmente, existe controversia respecto del rol de esta bacteria como agente causal de este tipo de patologías ${ }^{8-10}$. Escherichia coli es actualmente, junto a Streptococcus agalactiae, un patógeno frecuentemente aislado en sepsis neonatal y meningitis de aparición temprana ${ }^{11}$, siendo responsable, en alguna casuística, de $41 \%$ de las sepsis de aparición temprana en recién nacidos prematuros de muy bajo peso al nacer ${ }^{12}$. Por otra parte se ha evidenciado que esta bacteria ha sido aislada, a menudo, desde infección vaginal en cultivos polimicrobianos, asociada a reconocidos agentes de infección vaginal, y también desde cultivos monomicrobianos ${ }^{13,14}$.

Esta especie microbiana es un habitante normal del tracto gastrointestinal de animales y humanos. Es capaz de colonizar al hospedero sin causar enfermedad, produciéndose así un balance con el sistema inmune ${ }^{15,16}$. Estudios sobre la microbiota normal vaginal han determinado que E. coli no es un miembro de la microbiota del ecosistema vaginal $^{17}$.

Durante su co-evolución con el ser humano, E. coli ha desarrollado una gran capacidad para generar combinaciones de genes de virulencia aumentando su capacidad para colonizar diferentes hábitats de la anatomía humana. Actualmente se acepta que existen dos grupos de variedades patogénicas de E. coli. Los asociados a infecciones diarreogénicas y aquellos que ejercen su patogenicidad de modo extra-intestinal. Es así que se reconocen diversos patotipos diarreogénicos y unos pocos extra-intestinales ${ }^{18,19}$. Muchos de los genes de virulencia que han contribuido a la generación de estos patotipos se encuentran codificados en plásmidos, transposones y fagos, generando islas de patogenicidad específicas de cada uno de ellos ${ }^{20}$.
Universidad de Talca, Chile. Facultad de Ciencias de la Salud Departamento de Microbiología

No existen conflictos de interés. Esta investigación fue financiada por la Dirección de Investigación de la Universidad de Talca a través del proyecto VAC 600500.

Recibido: 30 de agosto de 2012 Aceptado: 11 de junio de 2013

Correspondencia a: Carlos Padilla Espinoza cpadilla@utalca.cl 
Es relevante investigar las cepas de E. coli aisladas desde cultivos monomicrobianos considerando que es el único agente aislado desde mujeres con síntomas de infección vaginal, pudiendo argüirse una responsabilidad en esta patología. Objetivos de este estudio fueron caracterizar genéticamente, mediante electroforesis en gel de campo pulsado (EGCP), 146 cepas de E. coli aisladas a partir de cultivos monomicrobianos obtenidos desde mujeres con síntomas de infección vaginal y determinar la presencia de genes de virulencia de cepas extra-intestinales.

\section{Material y Métodos}

\section{Cepas bacterianas y estudio microbiológico}

Fueron investigadas 146 cepas de $E$. coli de origen vaginal pertenecientes a la colección del Laboratorio de Investigación Microbiológica de la Universidad de Talca. Para calcular el tamaño de muestra requerido se consideró un intervalo de confianza de $95 \%$ con un error de precisión de $5 \%$ y una proporción estimada de $23 \%{ }^{13,24}$. En base a lo anterior, se estimó un máximo de 131 muestras (cepas). Considerando una pérdida eventual de $10 \%$, el número de muestras se incrementó a 146. Originalmente, las cepas fueron obtenidas desde cultivos monomicrobianos de flujos vaginales de mujeres con diagnóstico clínico de infección vaginal consultantes en el Hospital Regional de Talca. Este diagnóstico se realizó mediante exploración genital, pudiendo evidenciarse irritación e inflamación de la vulva acompañada de dolor en el coito y secreción vaginal.

\section{Aspectos éticos}

Todas las mujeres fueron informadas de los objetivos del estudio durante su chequeo ginecológico, obteniéndose un consentimiento informado previo a la toma de muestra. El protocolo de estudio fue aprobado por el Comité de Bioética de la Universidad de Talca.

\section{Criterios de inclusión}

Mujeres no embarazadas, no fumadoras, sin antecedentes de infecciones de transmisión sexual y con sólo una pareja sexual en los últimos 6 meses.

\section{Criterios de exclusión}

Haber recibido antimicrobianos en los seis meses previos al estudio, estar menstruando durante el examen clínico, estar cursando un cuadro diarreico o infecciones del tracto urinario (ITU).

El estudio y aislamiento original de las cepas de E. coli incluyó además el análisis de otros agentes infecciosos asociados a infección vaginal. Para ello la muestra vaginal fue recolectada de los fondos de saco lateral y posterior con tres tórulas estériles, las que se utilizaron en la ejecución de los test de Nugent y Amsel, además de una obser- vación directa, para detectar células micóticas. El estudio microbiológico de los otros microrganismos fue realizado de acuerdo a lo descrito en la literatura científica ${ }^{13,14}$. Las cepas de $E$. coli aisladas desde cultivos monomicrobianos fueron mantenidas en leche descremada $(20 \%)$ a $-70{ }^{\circ} \mathrm{C}$ hasta su utilización.

\section{Caracterización por electroforesis en gel de campo pulsado}

El protocolo de EGCP se basa en las recomendaciones de Maslow y cols. $(1993)^{21}$, modificado para este estudio. Cepas de E. coli fueron cultivadas por separado en 10 $\mathrm{ml}$ de caldo cerebro corazón (BHI) (Merck, Darmstadt, Alemania) durante aproximadamente ocho horas a $30^{\circ} \mathrm{C}$. Posteriormente, los cultivos fueron centrifugados a 3.000 g por 10 min a $4{ }^{\circ} \mathrm{C}$. Los precipitados obtenidos se lavaron dos veces con $\mathrm{NaCl} 1 \mathrm{~mol} / \mathrm{l}$ y Tris- $\mathrm{HCl}$ 0,01 mol/l, luego fueron re-suspendidos en Tris-HCl10 mmol/1 y EDTA 0,1 $\mathrm{mmol} / \mathrm{l}$. El ADN genómico fue preparado en cubos de agarosa mediante la mezcla de $0,5 \mathrm{ml}$ de suspensión de células bacterianas del precipitado más $0,5 \mathrm{ml}$ de agarosa de bajo punto de fusión al 2\% (Sigma-Aldrich, Inc., St. Louis, MO). Los cubos obtenidos fueron incubados durante toda la noche a $54{ }^{\circ} \mathrm{C}$ en tampón de lisis [Tris- $\mathrm{HCl}$ $50 \mathrm{mmol} / \mathrm{l} \mathrm{pH} \mathrm{8,0,} \mathrm{EDTA} 50$ mmol/1, 1\% lauryl-sarcosina, $1 \mathrm{mg} / \mathrm{ml}$ de proteinasa K (Sigma-Aldrich). Se lavaron tres veces por $30 \mathrm{~min}$ en agua destilada estéril y dos veces por 30 min en tampón TE (Tris-HCl10 mmol/1 pH 8,0; EDTA $1 \mathrm{mmol} / \mathrm{l}$ ) a $50^{\circ} \mathrm{C}$. Un pequeño trozo del cubo fue digerido durante la noche con $50 \mathrm{U}$ de $\mathrm{XbaI}$ (Fermentas, Hanover, Maryland) de acuerdo a las instrucciones del fabricante ${ }^{22}$. Los fragmentos resultantes se resolvieron mediante EGCP en agarosa al 1\% SeaKemGold (Cambrex, Cambridge, MA.) usando un aparato de campo pulsado CHEF-DR II (Bio-Rad Laboratories, Hercules, CA). Como marcador de tamaño molecular fue utilizado Lambda Ladder PFG (New England BioLabs Inc, Beverly, MA.). La electroforesis se realizó en 2,5 litros tampón TBE $0.5 \mathrm{X}$ durante $23 \mathrm{~h}$ a $6 \mathrm{~V} / \mathrm{cm}^{2}$ con un switch de tiempo de 1 a $30 \mathrm{~s}$, y con una temperatura del tampón de $14^{\circ} \mathrm{C}$. Los perfiles resultantes fueron comparados mediante el uso del software BioNumerics 3.0 (Matemáticas Aplicadas, de Saint-Martens-Latem, Bélgica), utilizando el método de grupo de pares no ponderado con la media aritmética (UPGMA) y coeficiente de similitud de Dice.

\section{Preparación de $A D N$}

Las 146 cepas de E. coli se cultivaron a $37^{\circ} \mathrm{C}$ en 10 $\mathrm{ml}$ de caldo $\mathrm{LB}\left(\mathrm{DO}_{550} 2,5\right)$. El cultivo se centrifugó y el precipitado bacteriano obtenido fue utilizado en la extracción de ADN genómico. Para este procedimiento se utilizó el kit Quantum Prep Aquapure ${ }^{\circledR}$ (Bio-Rad) siguiéndose las instrucciones del proveedor. El ADN se almacenó a $4{ }^{\circ} \mathrm{C}$ hasta su utilización. 


\section{Detección de secuencias específicas de genes de virulencia $(\mathrm{GV})$}

La detección de diez genes que codifican factores de virulencia se realizó mediante reacción de polimerasa en cadena-RPC. Los genes estudiados se encuentran asociados a cepas de E. coli responsable de infecciones extra-intestinales, considerando el origen de su aislamiento y fueron los siguientes: hly ( $\alpha$-hemolisina), iucC (aerobactina), afa (adhesina afimbrial), fimH (pili tipo 1), $n e u C$ (antígeno capsular K1), sfa/foc (pili tipo S y fimbrias tipo 1C), cnfl (factor necrosante citotóxico), papC (pili tipo $\mathrm{P})^{23-25}$, usp (proteína uropatógena específica) ${ }^{26,27} \mathrm{e}$ ibeA (factor de invasión del endotelio cerebral) ${ }^{26,27}$. Cada gen se estudió en las 146 cepas. Las secuencias oligonucleotídicas utilizadas se describen en la Tabla $1^{23,24,30}$. La metodología utilizada para la amplificación de los genes $h l y, i u c C$, afa, neuC, sfa/foc, fimH, papC, usp e ibeA, fue la siguiente: en un tubo Eppendorf se adicionaron $25 \mu \mathrm{l}$ de mezcla de reacción, tampón RPC $1 \mathrm{X}, 0.1 \mathrm{mM}$ de cada dNTP (Invitrogen, Carlsbad, CA.), $0.5 \mu \mathrm{l}$ cada secuencia oligonucleotídica, $0.5 \mathrm{U}$ de ADN Taq polimerasa (Invitrogen, San Diego, CA.) y $25 \mathrm{ng}$ de ADN templado. Las condiciones de reacción fueron una desnaturalización inicial a $94{ }^{\circ} \mathrm{C}$ por tres minutos, seguida de 25 ciclos de desnaturalización a $94{ }^{\circ} \mathrm{C}$ por un minuto, hibridación por un minuto a la temperatura de fusión de cada secuencia (Tabla 1), y extensión a 72 ${ }^{\circ} \mathrm{C}$ por un minuto, seguida de una extensión final de 10 minutos a $72{ }^{\circ} \mathrm{C}$. Para la detección y amplificación del gen $c n f 1$ se utilizó el siguiente protocolo: en un volumen total de $50 \mu$ que contienen $1 \mu 1$ de ADN, 900 ng de cada uno de los partidores específicos de cnfl, $\mathrm{MgCl}_{2} 1,5 \mathrm{mM}$, $0,2 \mathrm{mM}$ de cada dNTP y $1 \mathrm{U}$ Taq polimerasa. La secuencia de amplificación consistió en una desnaturalización a $94{ }^{\circ} \mathrm{C}$ por 2 minutos, seguida por 33 ciclos a $94^{\circ} \mathrm{C}$ por un minuto, $55^{\circ} \mathrm{C}$ por un minuto y $72{ }^{\circ} \mathrm{C}$ por un minuto, con una extensión final a $72{ }^{\circ} \mathrm{C}$ por dos minutos ${ }^{31}$. La amplificación de todos los genes se realizó en un termociclador DNA Engine ${ }^{\mathrm{TM}}$ (BioRad). Los productos de amplificación fueron separados por electroforesis en un gel de agarosa al $1,5 \%$ y posteriormente visualizados con bromuro de etidio $(0,25 \mu \mathrm{g} / \mathrm{ml})$. Un marcador molecular de ADN de $100 \mathrm{pb}$ (New England BioLabs Inc) fue utilizado en cada gel. Se adicionó un control negativo sin ADN. Todos los ensayos fueron realizados en duplicado.

\section{Análisis estadístico}

La correlación entre las distintas combinaciones de genes de virulencia y los grupos EGCP fue calculada utilizando el test de $\chi^{2}$ o test Exacto de Fisher (programa estadístico SPSS 15.0, IBM Statistics), según correspondiera. Un valor $\mathrm{p}<0,05$ fue considerado estadísticamente significativo para indicar la asociación entre ambas variables.

\section{Resultados}

Efectuada la EGCP se obtuvo en cada cepa de $E$. coli un promedio de 17 diferentes bandas con un rango variable entre 50 y $1000 \mathrm{~kb}$ (Figura 1). Cada una de las 146 cepas estudiadas presentó su respectivo perfil de EGCP. El análisis de perfiles de EGCP realizado por el método UPGMA y coeficiente de Dice, reveló que las cepas de E. coli analizadas pertenecían a dos grupos genéticos principales: grupo I (n: 53) y grupo II (n: 93) (Figura 2). La similitud genética intra-grupos fue cercana a $95 \%$ y entre los grupos levemente inferior a $80 \%$. Como dato específico se determinó que las cepas ov4, ov9, ov30, ov35, ov57, ov111 y ov143 fueron genéticamente indistinguibles entre sí, lo mismo que las cepas ov13, ov15, ov25 y ov93.

La Tabla 2 muestra la frecuencia de detección de los $\mathrm{GV}$ en las cepas de E. coli estudiadas. Los genes cnfl y fimH estaban presentes en la mayoría de las cepas. En aproximadamente $97 \%$ de las cepas se detectó el gen cnfl y el gen fimHen en $89 \%$. El gen afa no fue detectado. La Tabla 3 muestra las distintas combinaciones de GV y

\begin{tabular}{|c|c|c|}
\hline Genes & Secuencia de partidores $\left(5^{\prime}-3^{\prime}\right)$ & $\operatorname{Tm}\left({ }^{\circ} \mathrm{C}\right)$ \\
\hline papC & $\begin{array}{l}\text { 5〉-GACGGCTGTACTGCAGGGTGTGGCG-3» } \\
\text { 5»-ATATCCTTTCTGCAGGGATGCAATA-3〉 }\end{array}$ & 63 \\
\hline hly & $\begin{array}{l}\text { 5'-AACAAGGATAAGCACTGTTCTGGCT-3' } \\
\text { 5'-ACCATATAAGCGGTCATTCCCGTCA-3' }\end{array}$ & 63 \\
\hline afa & $\begin{array}{l}\text { 5'-GCTGGGCAGCAAACTGATAACTCTC-3' } \\
\text { 5'-CATCAAGCTGTTTGTTCGTCCGCCG-3' }\end{array}$ & 65 \\
\hline incC & $\begin{array}{l}\text { 5'-AAACCTGGCTTACGCAACTGT-3' } \\
\text { 5'-ACCCGTCTGCAAATCATGGAT-3' }\end{array}$ & 55 \\
\hline $\mathrm{fimH}$ & $\begin{array}{l}\text { 5'-TGCAGAACGGATAAGCCGTGG-3' } \\
\text { 5'-GCAGTCACCTGCCCTCCGGTA-3' }\end{array}$ & 55 \\
\hline neuc & $\begin{array}{l}\text { 5'-AGGTGAAAAGCCTGGTAGTGTG-3' } \\
\text { 5'-GGTGGTACATTCCGGGATGTC-3' }\end{array}$ & 61 \\
\hline$s f a / f o c$ & $\begin{array}{l}\text { 5'-CTCCGGAGAACTGGGTGCATCTTAC-3' } \\
\text { 5'-CGGAGGAGTAATTACAAACCTGGCA-3' }\end{array}$ & 65 \\
\hline cnf1 & $\begin{array}{l}\text { 5'-GGCGACAAATGCAGTATTGCTTGG-3' } \\
\text { 5'-GACGTTGGTTGCGGTAATTTTGGG-3' }\end{array}$ & 55 \\
\hline usp & $\begin{array}{l}\text { 5»-ATGCTACTGTTTCCGGGTAGTGTGT-3» } \\
\text { 5»-CATCATGTAGTCGGGGCGTAACAAT-3〉 }\end{array}$ & 66 \\
\hline ibeA & $\begin{array}{l}\text { 5»-AGGCAGGTGTGCGCCGCGTAC-3〉 } \\
\text { 5»-TGGTGCTCCGGCAAACCATGC-3» }\end{array}$ & 55 \\
\hline $\begin{array}{l}\text { * } \alpha \text {-hem } \\
\text { antígen } \\
\text { (sfa/foc) } \\
\text { (proteín } \\
\text { endotel }\end{array}$ & $\begin{array}{l}\text { ina ( } h / y) \text {, aerobactina (iucC), adhesina afi } \\
\text { apsular } \mathrm{K} 1 \text { (neuC), fimbria tipo } 1 \text { (fimH), fim } \\
\text { ctor citotóxico necrosante ( }(\mathrm{f} f 1) \text {, papC (pi } \\
\text { ropatógena específica) e IbeA (factor de } \\
\text { erebral). }\end{array}$ & $\begin{array}{l}\text { rial (afa), } \\
\text { as S y F1C } \\
\text { po P), usp } \\
\text { Jasión del }\end{array}$ \\
\hline
\end{tabular}




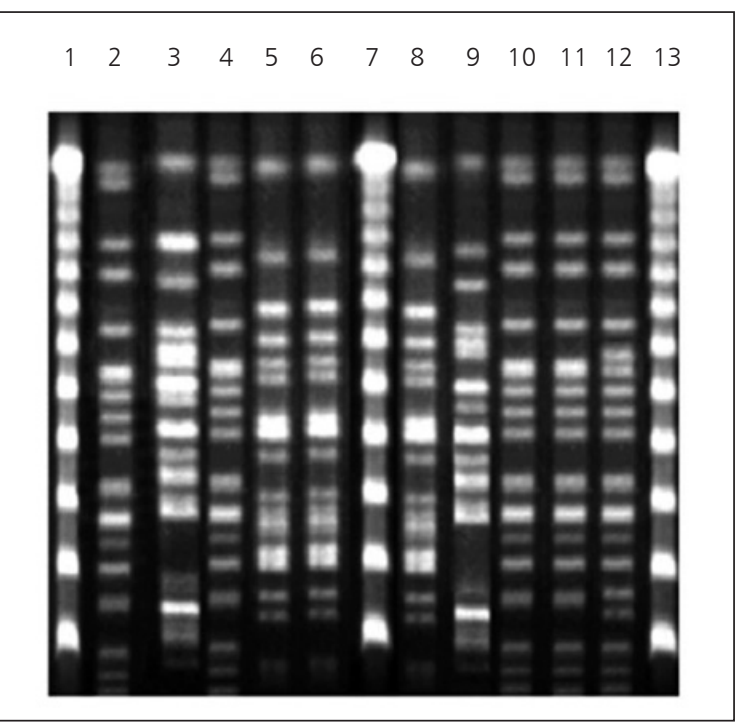

Figura 1. Perfiles de EGCP de E. coli proveniente de cultivos monomicrobianos de mujeres con infección vaginal. La digestión fue realizada con Xbal y analizada utilizando BioNumerics v. 3.0. Líneas 1, 7 y 13: marcador Lamda Ladder PFG N0340S; línea 2: cepa ov4; línea 3: cepa ov7; línea 4: cepa ov9; línea 5: cepa ov13:línea 6: cepa ov15; línea 8: cepa ov25; línea 9: cepa ov28; línea 10: cepa ov30; línea 11:cepa ov35; línea 12: cepa ov46.

Tabla 2. Frecuencia de detección de los diferentes genes de virulencia presentados por $E$. coli aislada desde infección vaginal (n: 146)

\begin{tabular}{|lrc|}
\hline Gen & $\mathbf{n} \mathbf{y} \% \mathbf{d e}$ cepas positivas \\
papC & 51 & $(34,9)$ \\
hly & 98 & $(67,1)$ \\
iucC & 89 & $(61,0)$ \\
afa & 0 & $(0)$ \\
neuC & 73 & $(50,0)$ \\
fimH & 130 & $(89,0)$ \\
sfa/foc & 79 & $(54,1)$ \\
cnf1 & 142 & $(97,3)$ \\
usp & 38 & $(26,0)$ \\
ibeA & 32 & $(21,9)$ \\
\hline
\end{tabular}

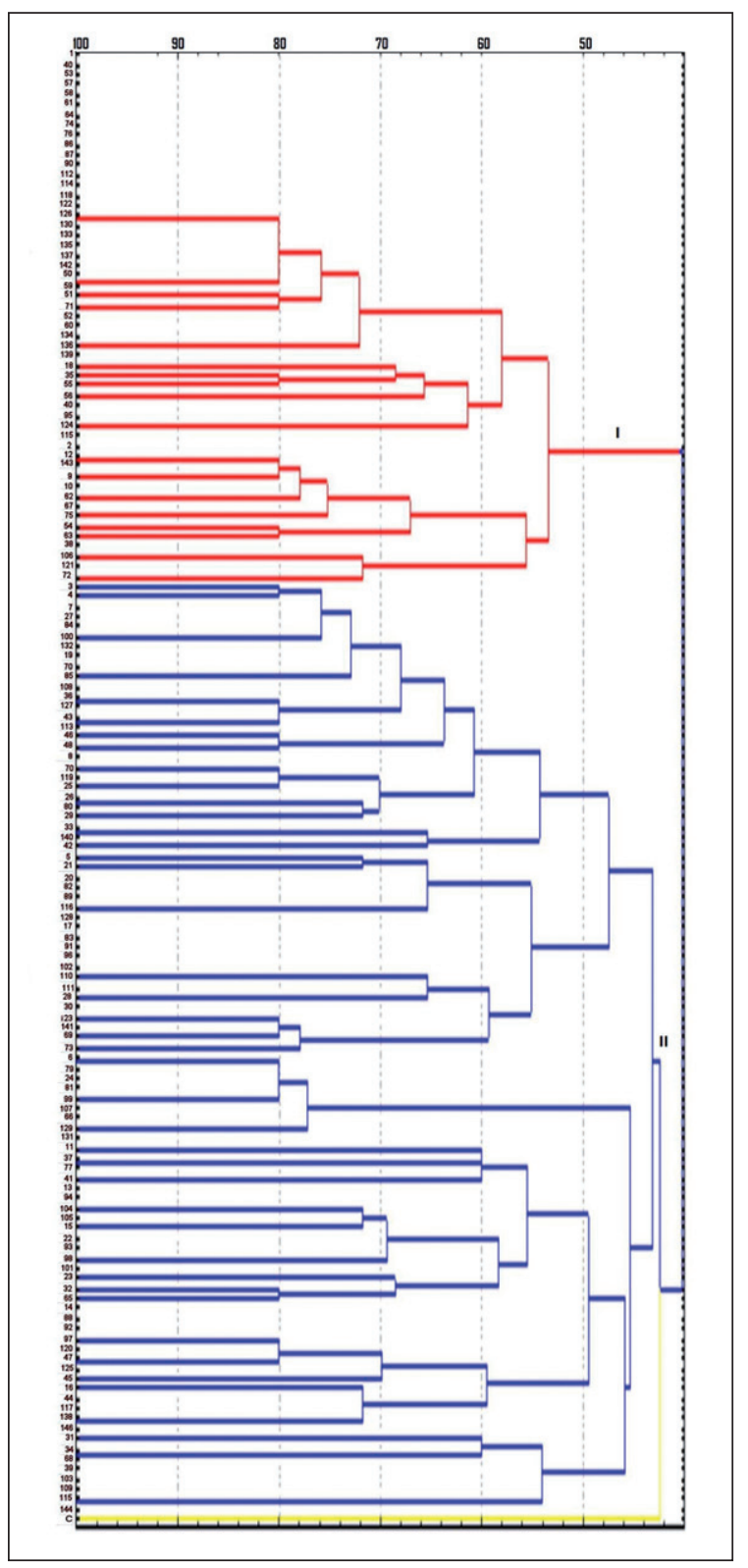

Figura 2. Dendrograma de perfiles de EGCP de 146 cepas de $E$. coli generado por el coeficiente de Dice y agrupado por UPGMA. La escala representa el porcentaje de similitud. C: control.

Tabla 3. Combinaciones de genes de virulencia (GV) en cepas de E. coli provenientes de cultivos monomicrobianos

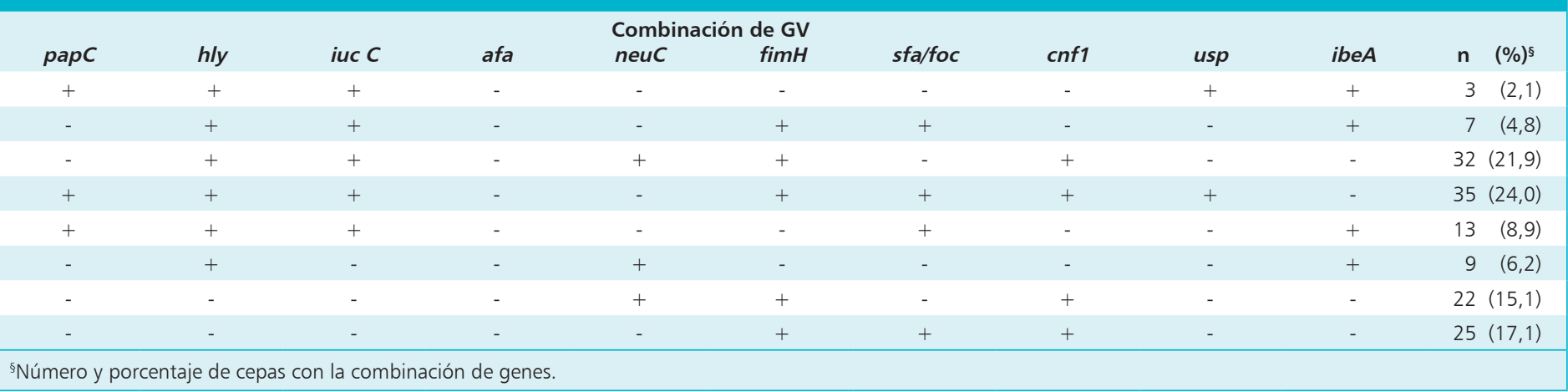


el número y porcentaje de cepas con cada combinación genética. Ocho combinaciones fueron determinadas en este estudio. La más común fue $p a p C+h l y+i u c C+a f a-$ $n e u C-f i m H+s f a / f o c+c n f 1+u s p+i b e A^{-}(\mathrm{n}: 35$ cepas $) . \mathrm{La}$ combinación menos frecuente fue papC + hly $+i u c C+$ afa- neuC-fimH-sfa/foc-cnfl-usp + ibeA+ (n: 3 cepas). No se observó una relación entre los perfiles genéticos (combinaciones) de los GV y los grupos originados a partir de los perfiles de EGCP detectados en este estudio.

\section{Discusión}

Actualmente, se estima que algunas cepas de E. coli pueden ser la causa de infecciones vaginales. En este trabajo se analizó a cepas de $E$. coli aisladas desde cultivos monomicrobianos, descartándose a cepas extraídas de cultivos polimicrobianos para eliminar responsabilidades patogénicas de las especies acompañantes, de reconocida capacidad para causar infecciones vaginales. No obstante, es probable que también entre estas cepas se encuentren variedades patogénicas poseedoras de factores de virulencia similares a los detectados en las cepas objeto de este estudio.

La EGCP se utiliza ampliamente como un método de sub-tipificación molecular de cepas de E. coli debido a su alto poder discriminatorio y su buena reproducibilidad ${ }^{32-34}$. En esta investigación, se utilizó EGCP por digestión con XbaI para comparar genéticamente 146 cepas de E. coli aisladas a partir de cultivos monomicrobianos desde mujeres con síntomas de infección vaginal. Los resultados demostraron que las cepas vaginales se distribuyen en dos grupos genéticos los que presentaron perfiles de EGCP similares. Ambos grupos mostraron un alto grado de similitud entre sus cepas. Estudios previos basados en la caracterización fenotípica y genómica de los polimorfismos de ADN (RAPD-PCR) revelaron que $E$. coli aislada desde mujeres con infección vaginal, eran parte de un único grupo genético, lo que implicaría una relación de parentesco entre ellas ${ }^{14}$. Los resultados de esta investigación, realizada con las cepas aisladas desde cultivos monomicrobianos utilizadas en los estudios anteriores ${ }^{13,14}$, permiten evidenciar de modo más certero la relación genética que presentan estas bacterias. Así, se ha logrado determinar la existencia de microorganismos genéticamente idénticos.

El estudio de los GV portados por las diferentes cepas bacterianas permite determinar su potencial patogénico. Así, cepas pertenecientes al mismo patotipo son genéticamente similares y portan los mismos $\mathrm{GV}^{19,35}$.

La presencia de GV en 146 cepas de E. coli estudiadas fue determinada por medio de RPC. Las cepas estudiadas de $E$. coli vaginales muestran un elevado número de factores de virulencia, lo cual les otorga un importante potencial de patogenicidad ${ }^{29}$. En general, la alta frecuencia de detección de los genes fim $H$ es relevante porque demuestra que las cepas de $E$. coli vaginales poseen características patogénicas similares a las de cepas uropatogénicas, en las cuales las fimbrias tipo 1 permanecen activas durante las etapas iniciales de la infección, facilitando la colonización genito-urinaria.

Se ha descrito que cepas portadoras de los genes codificantes del factor necrosante citotóxico (cnfl), fimbrias $\mathrm{P}$ ( papC) y hemolisina ( $h l y)$, están involucradas en infecciones extra-intestinales en seres humanos y animales $^{19,29}$. Estudios previos han demostrado que los genes del factor citotóxico necrosante y la toxina $\alpha$-hemolisina, son portados en la misma isla de patogenicidad (IP) ${ }^{36}$. En este estudio se detectó que $56 \%$ de las cepas presenta esta asociación, pudiendo argumentarse que las cepas de $E$. coli causantes de infecciones vaginales poseen algunas características de cepas necro-toxigénicas. También fue interesante observar que los GV más comunes son cnfl y fim $H$ seguido por los genes hly y iucC. Este último codifica el factor de virulencia aerobactina, cuya función es la absorción de hierro libre, lo que se considera un rasgo de virulencia importante ${ }^{37}$. De acuerdo con la literatura científica actual, la adhesina afimbrial codificada por el gen afa se encuentra en muy pocas cepas de $E$. coli ${ }^{31}$. En esta investigación ninguna de las cepas estudiadas presentó este gen, concordando con lo descrito en la literatura especializada en esta materia.

También se detectó que 50\% de las cepas eran portadoras del gen neu $C$ que codifica el antígeno K1, relacionado a meningitis neonatal ${ }^{38,39}$. Por otra parte, se ha observado una importante asociación entre cepas que codifican este antígeno y cepas productoras de septicemia que expresan fimbrias S y F1C (genes $s f a / f o c$ ). En el presente estudio se detectó $54 \%$ de cepas portadoras del gen sfa/foc y además, $22 \%$ presentó el gen de invasión del epitelio cerebral (ibeA). Cepas de E. coli con esta combinación de genes presentarían un alto nivel de virulencia en la eventualidad de un infección vaginal en mujeres embarazadas con el consiguiente riesgo de meningitis para el recién nacido ${ }^{24,40}$.

En esta investigación se encontraron diferentes combinaciones de GV en las cepas de estudiadas. Cada cepa presentó entre cuatro y cinco genes, lo cual revela un potencial de patogenicidad importante. La combinación de GV más frecuentemente detectada en este estudio presentó genes que codifican para fimbrias $\mathrm{P}$, tipo $1, \mathrm{~S}$ y F1C, además de hemolisina, factor citotóxico necrosante y proteína específica uropatógena. En este contexto, es importante destacar que esta combinación presenta el gen de $u s p$, cuya presencia indica el origen extra-intestinal de un número significativo de cepas de $E$. coli vaginales, demostrando al mismo tiempo una asociación de estas cepas con aquellas causantes de ITU. Desde un punto de vista clínico, no se pueden observar diferencias que permitan 
argüir la capacidad patogénica de cepas portadoras de un mayor o menor número de GV. Lo anterior debe asociarse también al comportamiento del genoma de estas cepas, en el cual la pérdida o ganancia de genes son procesos frecuentes. En este estudio, aun cuando se detectaron dos grupos genéticos mediante perfiles EGCP, no existió una correlación que permitiera asociar a cada uno de ellos con combinaciones específicas de GV.

En conclusión, este trabajo muestra que las cepas de $E$. coli aisladas desde infección vaginal a partir de cultivos monomicrobianos se concentran en dos grupos genéticos principales a partir de los perfiles de EGCP. Además se determinó que la mayoría de las cepas analizadas en este estudio, concentra un importante número de genes de virulencia presentes en $E$. coli aisladas de infecciones extra-intestinales. De este modo, resulta importante desarrollar nuevas estrategias de investigación en esta área, que profundicen el conocimiento sobre estas cepas y confirmar su verdadero rol en la infección vaginal.

\section{Resumen}

Introducción: Escherichia coli es capaz de producir diferentes cuadros infecciosos en el ser humano. Su patogenicidad en el tracto genital femenino es discutible. Objetivo: Determinar la presencia de genes de virulencia $(\mathrm{GV})$ en cepas de E. coli de procedencia vaginal. Material y Métodos: 146 cepas de E. coli aisladas desde infecciones vaginales a partir de cultivos monomicrobianos fueron estudiadas mediante EGCP y RPC. Los genes investigados fueron: $p a p C, h l y, i u c C$, afa, fimH, neuC, sfa/foc, cnfl, usp, e ibeA. Resultados: El análisis genético de las cepas demostró dos grupos con una similitud aproximada a $80 \%$ según Dice. La similitud genética intra-grupo fue aproximadamente de $95 \%$. Los resultados mostraron cepas con un alto número de $\mathrm{GV}$, siendo más comunes cnfl y fim $H$. El gen $a f a$ no fue detectado. Se determinaron ocho combinaciones de GV siendo la más común papC $+h l y+i u c C+a f a-n e u C-f i m H+s f a / f o c+$ cnfl+ usp+ibeA . Discusión: las cepas estudiadas se concentran en dos grupos genéticos característicos y la mayoría de las cepas analizadas concentra un importante número de $\mathrm{GV}$ presentes en $E$. coli aisladas de infecciones extra-intestinales. Conclusión: Es importante desarrollar nuevas estrategias de investigación en esta área, que permitan profundizar el conocimiento filogenético de estas cepas y confirmar su verdadero rol en la infección vaginal.

\section{Referencias bibliográficas}

1.- Kent H. Epidemiology of vaginosis. Am J Obstet Gynecol 1991; 165: 1168-72.

2.- Allen-Davis J, Beck A, Parker R, Ellis J, Polley D. Assessment of vulvovaginal complaints: accuracy of telephone triage and in-office diagnosis. Obstet Gynecol 2002; 99: $18-22$.

3.- Donders G. Definition and classification of abnormal vaginal flora. Best Pract Res Clin Obstet Gynaecol 2007; 21: 355-73.

4.- Gibbs R. The origins of stillbirth: infectious diseases. Semin Perinatol 2002; 26: 75-8.

5.- Goldenberg R y Thompson C. The infectious origins of stillbirth. Am J Obstet Gynecol 2003; 189: 861-73.

6.- Ghartey J P, Carpenter C, Gialanella P, Rising Ch, McAndrews T C, Mhatre M, et al. Association of bactericidal activity of genital tract secretions with Escherichia coli colonization in pregnancy. Am J Obstet Gynecol 2012; 207 (4): 297.

7.- Egan M E, Lipsky M S. Diagnosis of vaginitis. Am Fam Physician 2000; 62: 1095-104.

8.- Donders G G, Vereecken A, Bosmans E, Dekeersmaecker A, Salembier G, Spitz B. Definition of a type of abnormal vaginal flora that is distinct from bacterial vaginosis: aerobic vaginitis. Br J Obstet Gynaecol 2002; 109: 34-43.

9.- Weir E. Bacterial vaginosis: more questions than answers. Can Med Assoc J 2004; 171: 448.

10.- Forsum U, Holst E, Larsson G, Vásquez A, Jakobsson T, Mattsby-Baltzer I. Bacterial vaginosis-a microbiological and immunological enigma. APMIS 2005; 113: 81- 90.

11.- Lin C Y, Hsu C H, Huang F Y, Chang J H, Huang F Y, Chang J H, et al. The changing face of early-onset neonatal sepsis after the implementation of a maternal group B Streptococcus screening and intrapartum prophylaxis policy-a study in one medical center. Pediatr Neonatol 2011; 52: 78-84.

12.- Stoll B J, Hansen N I, Sánchez P J, Faix R G, Poindexter B B, Van Meurs K P, et al. Early onset neonatal sepsis: the burden of group $\mathrm{B}$ streptococcal and $E$. coli diseases continues. Pediatrics 2011; 127: 817-26.

13.- Padilla C, Lobos O, Padilla R, Fuentes L, Nuñez L. Aislamiento de cepas de Escherichia coli desde casos clínicos de infección vaginal: asociación con otros microorganismos y susceptibilidad antibacteriana. Rev Chil Obstet Ginecol 2007; 72: 222-8.

14.- Lobos O, Padilla C. Phenotypic characterization and genomic DNA polymorphisms of Escherichia coli strains isolated as the sole microorganism from vaginal infections. Microbiology 2009; 155: 825-30.

15.- Escobar-Páramo P, Grenet K, Le Menac'h A, Rode L, Salgado E, Amorin C, et al. Large-scale population structure of human commensal Escherichia coli isolates. Appl
Environ Microbiol 2004; 70: 5698-700

16.- Sasakawa C, Kacker J. Host-microbe interaction: bacteria. Curr Opin Microbiol 2006; 9: 1-4.

17.- Hyman R, Fukushima M, Diamond L, Kumm J, Giudice L, Davis, R. Microbes on the human vaginal epithelium. PNAS 2005; 31: 7952-57.

18.- Bielaszewska M, Dobrindt U, GärtnerJ, Gallitz I, Hacker J, Karch, H, et al. Aspects of genome plasticity in pathogenic Escherichia coli. Int J Med Microbiol 2007; 297: 625-39.

19.- Bekal S, Brousseau R, Masson L, Prefontaine G, Fairbrother J, Harel J. Rapid identification of Escherichia coli pathotypes by virulence gene detection with DNA microarrays. J ClinMicrobiol 2003; 41: 2113-25.

20.- Schmidt H, Hensel M. Pathogenicity islands in bacterial pathogenesis. Clin Microbiol Rev 2004, 17: 14-56.

21.- Maslow J, Slutsky A, Arbeit R. Application of pulsed-field electrophoresis to molecular epidemiology. In: Persing DH, Smith TF, Tenover FC, White TJ, editors.Diagnostic Molecular Microbiology Principles and Applications. Washington DC: American Society for Microbiology; 1993, p. 563-72.

22.- Ejrnaes K, Sandvang D, Lundgren B, Ferry S, Holm S, Monsen T, et al. Pulsed-field gel electrophoresis typing of Escherichia coli 
strains from samples collected before and after pivmecillinam or placebo treatment of uncomplicated community-acquired urinary tract infection in women. J Clin Microbiol 2006; 44: 1776-81.

23.- Le Bouguenec C, Archambaud M, Labigne A. Rapid and specific detection of the pap, afa, and $s f a$ adhesin-encoding operons in uropathogenic Escherichia coli strains by polymerase chain reaction. J Clin Microbiol 1992; 30: 1189-93.

24.- Watt S, Lanotte P, Meneghetti L, Moulin-Schouleur M, Picard B, Quentin R. Escherichia coli strains from pregnant women and neonates: interspecies genetic distribution and prevalence of virulence factors. J Clin Microbiol 2003; 41: 1929-35.

25.- Bonacorsi S, Houdouin V, Mariani-Kurkdjian P, Mahjoub-Messai F, Bingen, E. Comparative prevalence of virulence factors in Escherichia coli causing urinary tract infection in male infants with and without bacteremia. J Clin Microbiol 2006; 44: 1156-58.

26.- Kanamaru S, Kurazono H, Ishitova S, Terai A, Habuchi T, Nakano M, et al. Distribution and genetic association of putative uropathogenic virulence factors iroN, iha, $k p s M T$, ompT and usp in Escherichia coli isolated from urinary tract infections in Japan. J Urol 2003; 170: 2490-93.

27.- Marrs C, Zhang L, Foxman B. Escherichia coli mediated urinary tract infections: Are there distinct uropathogenicE. coli (UPEC) pathotypes? FEMS Microbiol Lett 2005; 252:
183-90.

28.- Takahashi A, Kanamaru S, Kurazono H, Kunishima Y, Tsukamoto T, Ogawa O, et al. Escherichia coli isolates associated with uncomplicated and complicated cystitis and asymptomatic bacteriuria possess similar phylogenies, virulence genes, and O-serogroup profiles. J Clin Microbiol 2006; 44: 4589-92.

29.- Hilbert D, Paulish T, Mordechai E, Adelson M, Trama J. O serogroups, phylogeny, and virulence factors of cervico vaginal and rectal Escherichia coli isolates. Eur J Clin Microbiol Infect Dis 2008; 27: 1265-8.

30.- Pass M, Odedra R, Batt R. Multiplex PCRs for identification of Escherichia coli virulence genes. J Clin Microbiol 2000; 38: 2001-04.

31.- Birosová E, Siegfried L, Kmetová M, Makara A, Ostró A, Gresová A, et al. Detection of virulence factors in $\alpha$-haemolytic Escherichia coli strains isolated from various clinical materials. Clin. Microbiol Infect 2004; 10: 569-73.

32.- Johnson J, Russo T. Molecular epidemiology of extra-intestinal pathogenic (uropathogenic) Escherichia coli. Intern J Med Microbiol 2005; 295: 383-404.

33.- Weiner M, Dacko J, Osek J. Molecular analysis of enterotoxigenic, shiga toxigenic and enteroaggregative Escherichia coli strains isolated from suckling piglets with diarrhoea by the use of pulsed-field electrophoresis. Bull Vet Inst Pulawy 2004, 48: 241-46.

34.- Welinder-Olsson C, Badenfors M, Cheasty T,
Kjellin E y Kaijser B. Genetic profiling of enterohemorrhagic Escherichia coli strains in relation to clonality and clinical signs of infection. J Clin Microbiol 2002; 40: 959-64.

35.- Croxen M, Finlay B. Molecular mechanisms of Escherichia coli pathogenicity. Nat Rev Microbiol 2010, 8: 26-38.

36.- Ruiz J, Simon K, Horcajada J, Velasco M, Barranco M, Roig G, et al. Differences in virulence factors among clinical isolates of Escherichia coli causing cystitis and pyelonephritis in women and prostatitis in men. J Clin Microbiol 2002; 40: 445- 9 .

37.- Gordon D, O’Brien C. Bacteriocin diversity and the frequency of multiple bacteriocin production in Escherichia coli. Microbiology 2006; 152: 3239-44.

38.- Obata-Yasuoka M, Ba-Thein $\mathrm{W}$, Tsukamoto T, Yoshikawa H, Hayashi, H. Vaginal Escherichia coli share common virulence factor profiles, serotypes, and phylogeny with other extraintestinal E. coli. Microbiology 2002; 148: 2745-52.

39.- Kaper J, Nataro J, Mobley T. Pathogenic Escherichia coli. Nat Rev Microbiol 2004; 2: 123-40.

40.- Martínez-Medina M, Mora A, Blanco M, López C, Alonso M, Bonacorsi S, et al. Similarity and divergence among adherentinvasive Escherichia coli and extra-intestinal pathogenic E. coli strains. J Clin Microbiol 2009; 47: 3968-79. 